\title{
FUNCION DE LOS OBJETOS EN EL IDILIO III DE TEÓCRITO
}

\author{
Maximo F. Borghini \\ Universidad de Buenos Aires
}

Resumo: El Idilio III, que se inscribe dentro de las composiciones bucólicas de Teócrito, es a la vez un paraklausAhyron rústico que tiene como protagonistas a un anónimo cabrero y a una invisible pastora llamada Amaryllis. El tema esencial, por consiguiente, gira alrededor del amor.

El Presente trabajo ha sido enfocado exclusivamente desde el plano de los objetos, una perspectiva de análisis que, por lo general, es poco habitual. El exámen arroja como resultado que tales objetos son escasos en la pieza, pero que todos ellos, debidamente catalogados y analizados uno por uno, están íntimamente relacionados con el tema dominante del poema, y que su estudio nos ayuda a complementar las vicisitudes amorosas del cabrero.

El Idilio III de Teócrito es un poema bucólico íntegramente conformado por el monólogo de un anónimo cabrero, enamorado de la pastora Amaryllis.

Si repasamos por un momento el asunto, recordaremos que el cabrero confla su rebaño a un ayudante 0 a un pastor colega (vv. 1-5) para dirigirse hacia la cueva de Amaryllis frente a la cual ensayará un extenso canto amoroso (vv. 6-51). Desde este punto de vista, el poema puede ser definido como un verdadero paraklausíthyron, si bien con características peculiares.

El tema predominante de la composición, por lo tanto, gira alrededor del amor, no infrecuente en el poeta de Siracusa, quien suele realzarlo a través de una rica y compleja trama de motivos.

El propósito del presente trabajo es focalizar el análisis desde la perspectiva del plano de los objetos ${ }^{1}$ y determinar su incidencia en la economía del idilio.

\section{Los Objetos Reales}

En nuestro texto el protagonista integra de hecho un solitario komos (v. 1) cuya meta es la gruta de la amada.

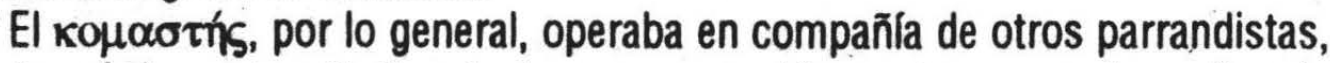
a menudo tan ebrios como él. Iban juntos, en procesión nocturna, por las calles de

1. Se entiende aqui por objotos el conjunto de "cosas" que no pertececen al plano estrictamente vegetal ni constituyen "elementos de paisaje" (ríos, montañas, etc.). 
la ciudad, coronados de guirnaldas, portando antorchas y, en ocasiones, una serie de utensilios para quebrar los cerrojos más obstinados².

Pero deciamos que el presente paraklausíthyron posee características especiales: el espacio y el ambiente no son urbanos, como es de esperarse, sino agrestes; no hay mención de vino ni de ebriedad; las antorchas son innecesarias porque la acción transcurre de día, y la puerta, en tanto construcción material, no existe ya que la entrada a la cueva está cubierta exclusivamente de hiedras y helechos (v. 14). De todos los implementos habituales del amator, sólo sobrevive la guirnalda.

La guirnalda o corona ( $\sigma \tau \varepsilon \varphi \alpha$ vos) solía ser colgada a la puerta de la persona amada como ofrenda amorosa, y es con esta simbología que aparece en los vv. 21-23 del Idilio III. No es el primer don mediante el cual el cabrero tienta a una muda e impasible Amaryllis: antes fueron las diez manzanas recolectadas en un sitio escogido (vv. 10 ss.), y luego vendrá el ofrecimiento de una cabra del rebaño, fértil y blanca - tal vez lo más preciado que el personaje sea capaz de entregar - pero que, de todos modos, quedará en otras manos menos pretenciosas (vv. 34 ss.).

La guirnalda, mencionada a comienzo de verso y del entero pasaje en clara posición de relieve, representa un hito palpable de una suerte de "itinerario del dolor" del cabrero, que comienza con la constatación de la indiferencia de Amaryllis (vv. 7 ss:) y con ese penetrante óx $\chi 0 s$ del v. 12, para prolongarse en el motivo de la crueldad de Eros (vv. 15 ss.) y en la falsa alegría de unos besos vacíos (vv. 19 ss.).

Sobre la corona en cuestión se pueden inferir, a partir de nuestro pasaje, los siguientes datos:

- la guirnalda está en poder del cabrero, quien amenaza con destruirla. Dicha amenaza que, en cuanto tal, apunta por lógica a un hecho aún no consumado, gira alrededor de una idea de futuro señalada por


son frecuentes y que la mayoría de ellos expresa la probable toma de decisiones que, de verificarse, se revelarían negativas para la salud física y espiritual del hablante ${ }^{3}$.

- la amenaza de deshojar la guirnalda ( $\tau \hat{\imath} \lambda \alpha \imath$ ) hasta reducirla al esqueleto ( $\kappa \alpha \tau \dot{\alpha} \lambda \varepsilon \pi \tau \alpha \alpha)$ constituye el preanuncio del motivo del suicidio que será formulado en seguida después (vv. $25 \mathrm{ss}$.).

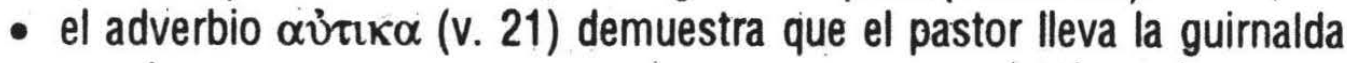
consigo.

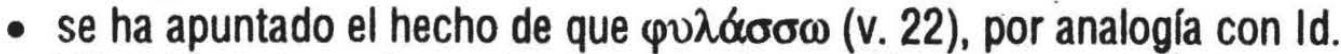
VII, 64 señale aquí que el cabrero la tenga ceñida a su cabeza ${ }^{4}$. Pero el verbo no puede distar mucho en significado e intencionalidad del

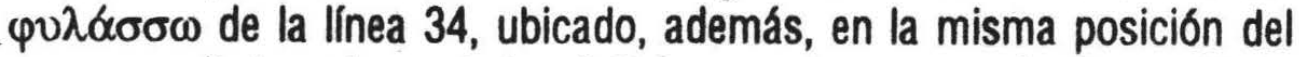
verso: aquil, la cabra criada afectuosamente por su dueño y alli, la

2. Cfr. H. Canter, "The paraclausfthyron as a literary theme", NPh 41, (1920), 355-368. C. Soria, "EI

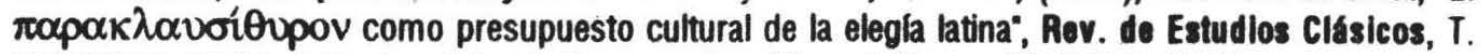
VIII (1963), 55-94. J. Yardley, "The elegiac paraclausfithyron", Eranos 76 (1978), 19-34.

3. Cfr. w. 9, 25, 36, 53; cfr. también vv. 11, 37, 38 y 51.

4. Cfr. A. Gow, Theocritus, Cambridge 1952, II, p. 69. 
corona entretejida con esmero, son por igual dones de amor por los cuales el amante procura obtener alguna respuesta favorable de parte de Amaryllis.

- la presencia del pronombre de primera persona ( $(\dot{\varepsilon} \gamma \omega v, v .22)$ enfatiza

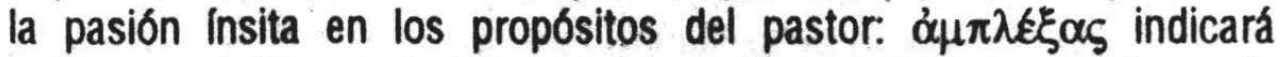
entonces, el diligente trabajo de elaboración del objeto y $\varphi v \lambda \alpha \alpha \sigma \sigma \omega$ la custodia celosa de la guirnalda terminada a la espera del momento propicio para donarla.

- la composición de la corona responde también a un cuidado que se origina en el amor del cabrero por Amaryllis.

La base, en este caso, está formada de ramas y hojas de hiedra ya que es menos probable la interpretación de quien relaciona el genitivo ikioooto con

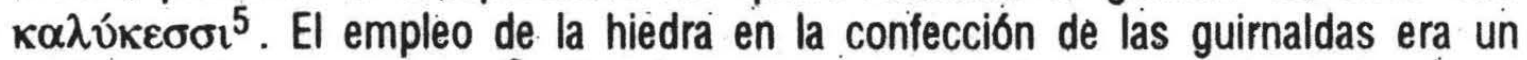
hecho corriente y habitual ${ }^{6}$. En el terreno religioso, la corona de hiedra es un atributo asociado, desde los trágicos y en el propio Teócrito ${ }^{7}$, a Dionisio y su culto, atributo que se transmite también a alguno de los acompañantes de este dios, como por ejemplo, Priapo $^{8}$.

$K \alpha \hat{\lambda} \nu \xi$, propiamente es el capullo de una flor que, de ordinario y en nuestro pasaje, es la rosa, según ya anticipan los escolios ${ }^{9}$. La rosa es un tradicional símbolo erótico. Sin ir más allá del corpus teocriteo, baste aquí recordar la desbordante pasión de Polifemo, quien no se limita a amar a Galatea con manzanas, bucles o rosas, es decir las prendas de amor más convencionales ${ }^{10}, 0$ también el canto de Buceo a su amada, en el que se relaciona la rosa con Afrodita ${ }^{11}$.

La presencia del apio nos sugiere de immediato las coronas trenzadas para los vencedores de los juegos ístmicos y de Nemea ${ }^{12}$, pero también era utilizado en el culto de Dionisio y en las orgĺas báquicas ${ }^{13}$. El apio, en Teócrito, es una especie apreciada, destacada por su exuberancia, su sinuosidad ${ }^{14} 0$, como en el Idilio III, por su aroma. Especlficamente, el aroma del apio era valorado por sus cualidades y efectos refrescantes asI como por el hecho de ahuyentar animales molestos o venenosos ${ }^{15}$.

Pero el apio no es sólo símbolo de victoria, sino también un ingrediente para reanimar a los moribundos y hallable, entonces, en los momentos de duelo y

5. Scholia in Theocritum, p. 29.

6. Plinio, N. H., 21, 52.

7. Eur. Bacch. 81, 702; Soph., Tr. 220; Theocr., XXVI, 4.

8. Theocr. Epigr. 3, 3; K. Lembach, Die Plianzen bel Theokrit, Heidelberg, 1970, pp. 118 ss.

9. 8chol. in Theoer., p. 29.

10. Theocr., XI, 10.

11. Theocr., $X, 33$ ss.

12. Ctr. Pindaro, 01, XIII, 46, Nem. N, 143, etc.

13. K. Lembach, op. ch., pp. 34 ss.

14. Theocr., VII, 68; XIII, 42.

15 . K. Lembach, op. elt., pp. 34 ss. 
de dolor ${ }^{16}$. No sería extraño, pues, advertir este eco simbólico de infelicidad,

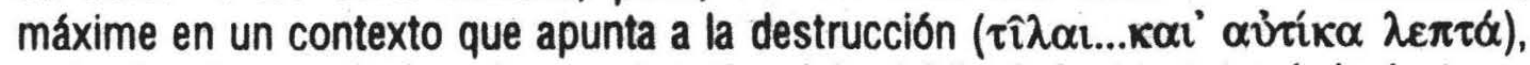
y si, además, es relacionado con el motivo del suicidio de los versos subsiguientes 0 con el final del idilio en que se anuncia la oupovití $\alpha$ del cabrero.

El otro objeto de existencia concreta en el presente del idilio, es la casaca del pastor, y está incluído en aquel motivo que hemos mencionado como la amenaza de suicidio (vv. 25 ss.).

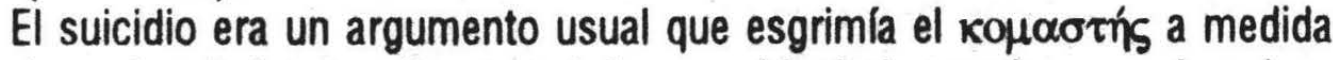
que perdía la paciencia frente a la puerta infranqueable de la amada, y en el cual no había que creer demasiado. Aquil tampoco habrá que tomar las palabras del cabrero al pie de la letra.

Boítn indica una zamarra hecha de piel de animal (cabra, carnero y símiles) usada por los pastores y los campesinos de las regiones del Mediterráneo. El escoliasta explica el término mediante transparentes sinónimos como el bien

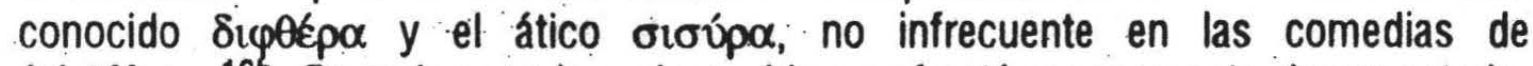
Aristófanes ${ }^{160}$. De todos modos; el vocablo en sl está escasamente documentado. Lo conoce Herodoto, que lo menciona al describir las costumbres escitas ${ }^{17}$ y lo que aún es más importante, está presente en los mimos de Sofrón, admirados e

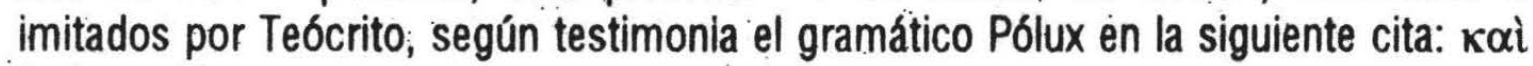

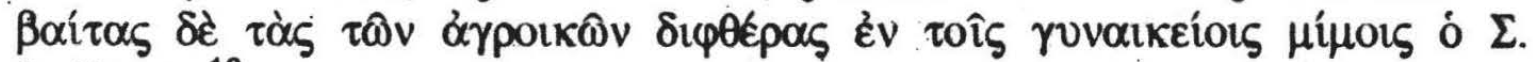

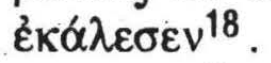

En nuestro pasaje, ßaírn, que nos devuelve de modo inequívoco al

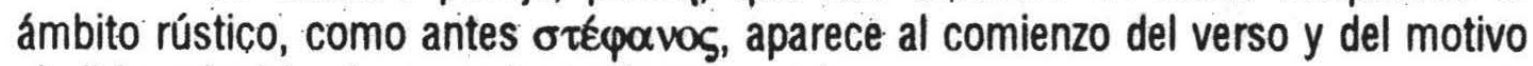
aludido, adquiriendo una relevancia no casual.

Estos versos, por semejanza de estructura y de núcleos semánticos, tienen una estrecha similitud con los vv. 15-16 del Idilio V: también aquil hay un pastor que, acusado de robo, anuncia que se arrojará a las aguas del Krathis desde 10 alto de una roca; y también aquil convendira dudar de tales afirmaciones. La diferencia esencial entre ambos textos estriba en la motivación de este "salto a las aguas".

En el Idilio III el cabrero predice que se lanzará al mar desde donde el pescador Olpis espía a los atunes (v. 26). Esta aserción es explicable a partir de las Haliéuticas de 0 piano ${ }^{19}$, quien expone una técnica de los pescadores consistente en la atenta observación de las aguas desde un lugar elevado por parte de uno de ellos. Este, mediante señas, informa a sus compañeros, ubicados en otra posición, acerca del desplazamiento de los peces. En otras palabras, la mención de Olpis y de los atunes ayuda a definir la insinuación del cabrero, es decir, a entender que él se arrojará desde el peñasco más alto.

Ahora bien, para quien toma una determinación de tal magnitud, precipitarse a las aguas con o sin casaca, la diferencia es insignificante. Pero el

16. scol. In Theocr., p. 29; Herod. IV, 71.

16b. Schol. in Theocr., p. 29.

17 . Herod. IV, 64.

18. Sophr. 38 en Comicorum Graecorum Fragmenta, Ed. Kaibel, I, Berlin (1958).

19. 0. Hal. III, 637, citado por Gow, op. ch., II, 69. 
énfasis y la relevancia que, dijimos, se le da a ßaírn, y la repetición de la idea en Id. $V, 15-16$, parece indicarnos un pequeño ritual: el hecho de despojarse del chaleco, distintivo del cabrero, señalaría la pérdida de un objeto fiel, de un símbolo que se constituye en razón de vida del pastor. Perecer sin la zamarra aumentaría la crueldad de la muerte. En otro tono, incluso Polifemo entregarla algo característico de si - el único ojo - con tal de resolver favorablemente sus cuestiones amorosas ${ }^{20}$.

El motivo de la muerte voluntaria aparece en otros momentos de la composición: la muerte por ahorcamiento (v. 9) que llama a la memoria el asunto del Idilio XXIII y la muerte - como se verá más adelante - bajo las fauces de los lobos (vv. 53-54). La sugerida muerte en las aguas es una realidad para otros personajes protagónicos de Teócrito, como Dafnis o Hylas. En el caso del cabrero, es sólo una bravuconada.

El verso 27 - en la mitad del poema - junto al verso 54 , que cierra la pieza, destacan la insensibilidad completa de Amaryllis, para quien el mal fin del cabrero sería un dulce placer, como lo sugieren los términos $\alpha \delta \delta$ / $\mu \varepsilon \lambda_{l} / \gamma \lambda v \kappa v$. Este verso, además, concluye en clímax ese mencionado "itinerario del dolor" durante el cual el personaje libra y pierde la batalla ante la indiferencia de la pastora. De aquí en más, el cabrero recordará instantes pasados en la historia de su pasión, y en la segunda parte del canto citará célebres amores de la mitología.

En conclusión, los w. 25-27 desarrollan el tópico del suicidio que el exclusus amator solía exponer en algún punto del lamento frente a la puerta cerrada. El propósito, por supuesto, no era concretar la amenaza sino conmover a la amada.

La zalea, es decir el objeto enfatizado en la pretendida última acción en vida del hablante, trasciende hacia un plano simbolico en el que, por un lado, el pastor de cabras se diferencia en su unicidad de cualquier otro tipo humano sobre la tierra, y por el otro, realza la propia zamarra como el único elemento capaz y merecedor de subsistir a la destrucción.

\section{Los Objetivos Evocados}

Un caso distinto es el de los objetos que son traídos a colación no por su presencia material sino como indicadores evidentes de otras realidades.

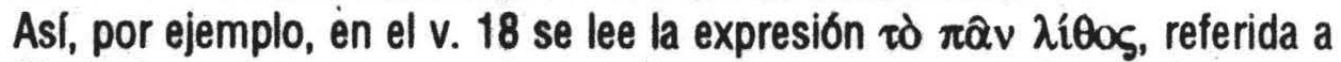
Amaryllis. El sintagma integra un vasto vocativo que se corresponde con los otros dos, más sencillos y espontáneos, de los versos 6 y 22, en los que la ninfa es

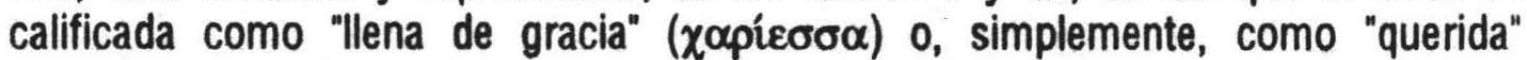
$(\varphi i \lambda \alpha)$. El vocativo del v. 18, en cambio, es más elaborado y consta de tres miembros, dos de los cuales, el primero y el tercero, están de alguna manera inspirados en los ojos y la mirada de la joven. En el centro, $\tau \grave{\partial} \pi \hat{\alpha} v \nu i ́ \theta 0 s$ abarca por entero la personalidad de Amaryllis.

La lección $\lambda i ́ \theta 0 s$, que deberá anteponerse a la variante $\lambda i ́ \pi \varsigma^{21}$, en especial por su aparición concorde en los manuscritos, aproxima a la amada al plano de los objetos. Y llamar a alguien $\lambda i ́ \theta 0 s$ era, y lo sigue siendo, una manera de

20. Theocr. VI, 53.

21. Schol. in Theocr., p. 29. 
resaltar su insensibilidad emocional y su dureza de espiritu22 . La sugerencia del escoliasta acerca de la ecuación $\lambda i ́ \theta 0 s=$ mármol $^{23}$ implica que la imagen apunta a la blancura de la piel de la muchacha, color atractivo para los pastores bronceados por el fuerte sol mediterráneo. Pero el término, sin algún atributo alusivo como $\lambda \varepsilon v$ Kó $_{\mathrm{s}} \mathrm{u}$ otros semejantes ${ }^{24}$, en sentido absoluto no significa que deba equivaler a "mármol".

Queda en pie, pues, la tesis de la insensibilidad. La objeción acerca de la contradicción de un calificativo agresivo en medio de otros dos encomiásticos, es relativa: éstos reconocen la belleza en los ojos de Amaryllis, pero $\lambda i ́ \theta o s$ la define de una pieza. Juntas, además, la mirada y la piedra, recuerdan el mito de la Gorgona.

Si se acepta, entonces, leer $\tau \grave{\partial} \pi \hat{\alpha} v \lambda i \theta_{0 s}$, hallaremos que un objeto no presente 0 , al menos, irrelevante en el plano real del idilio, se proyecta simbólicamente hasta alcanzar resonancia y sentido en el nivel de la afectividad humana. La fórmula tò $\pi \hat{\alpha} v$, con valor adverbial, acentúa este simbolismo de $\lambda i ́ \theta 0 s$.

El próximo objeto se desoculta de un modo diferente.

Decíamos que, en el v. 27 - mitad del poema - el cabrero abandona la táctica directa en la conquista de la amada, y que, de alli en adelante, concluída la parte más dinámica del idilio, se internará en una inoperante vía mitológica (vv. 4051), no sin antes detenerse en algunos momentos claves del pasado inmediato que anticipan su derrota final. Uno de tales momentos pone en relieve la figura de

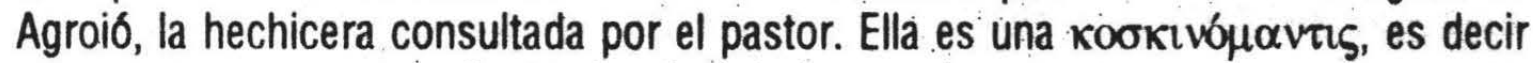
una maga que opera mediante el cedazo (Kóokıvov).

Los temas mágicos han interesado en la antigüedad a diversos autores, y Teócrito, sin duda, se encuentra entre ellos: baste pensar en el Idilio II.

Los estudiosos modernos, incluído Gow ${ }^{25}$, no hallando información fidedigna en los textos antiguos, ni siquiera en los escolios del corpus teocriteo, no han podido explicar la práctica de la criba. Sólo Arnott ha sabido echar un poco más de luz sobre la cuestión a partir de un pasaje de una novela de Kazantzakis ${ }^{26}$. En ella, Kazantzakis describe la hechicería del cedazo situándola en la Creta de fin de siglo. El uso, atestiguado casi de modo lacónico en el texto, se habría perpetuado entre los pobladores de las islas griegas hasta los tiempos modernos.

Aqul, por el momento, nos interesa rescatar del término кoбкıvó $\mu \alpha v \tau \iota s$

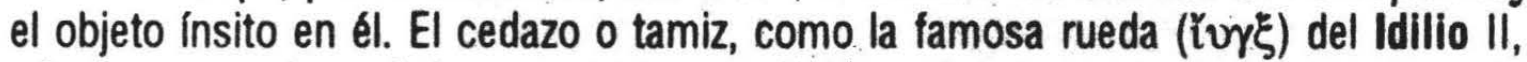
adquiere un valor mágico $y$, en consecuencia, se le otorga un radio de influencia sobre la vida cotidiana mayor del que podría ofrecer cualquier otro utensilio común.

En el texto, el tamiz es otro objeto que se relaciona con la historia amorosa del cabrero, esta vez por medio de la magia, y que tiene su repercusión en el plano de la credibilidad humana. Más que la propia Agroio, quien "habla" es el mismo cedazo, y su veredicto condiciona al amante en su espiritu y en su conducta, plegándolo lentamente hacia una inevitable resignación.

22. Gow, op. elt., II, p. 68; Theocr., XXIII, 20.

23. Schol. In Theocr., p. 29.

24. Theocr. VI, 38.

25. Gow, op. clt., II, p. 71.

26. G. Arnott, "Coscinomancy in Theocritus and Kazantzakis", Mnem. 31, Fasc. I (1978), 27-32. 


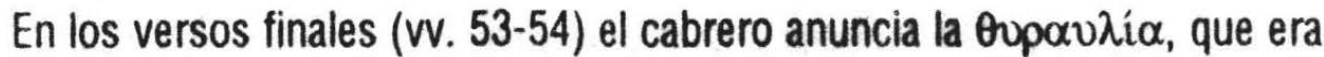
el intento extremo del amator por despertar en el ser amado un destello de compasión.

El hecho de tenderse sobre los umbrales de la cueva y la mención de los lobos anticipan, incluso con exageración, el momento de la noche que, en el presente del idilio, está aún lejos de concretarse. Con esta presunta muerte a merced de los lobos, que se relaciona, según dijimos, con el motivo de la amenaza de suicidio ( $v v .25$ ss.), el pasaje tiende a remarcar una vez más - desde la óptica del cabrero - la cruel insensibilidad de Amaryllis, cuyo peso central recae sobre la metáfora de la miel.

La miel, que, en lineas generales; posee dentro de la cultura griega, una connotación francamente positiva y que, en Teócrito en particular, deviene, sin apartarse de la tradición, el alimento de los poetas 27 , o bien es digna de figurar entre las ofrendas a $\mathrm{Pan}^{28}$, adquiere aquí un tinte fúnebre: su evocación lleva al extremo de lo agradable la verificación de la desaparición definitiva del cabrero.

Amaryllis, pues, es vista en el último verso bajo una luz siniestra, casi como si ella misma, loba entre los lobos, participara del imaginario festín nocturno a expensas de su enamorado.

El objeto-miel, sobre el final del ídilio, pasa a integrar el plano simbólico revistiendo características tétricas, sugeridas por una representación casi canibalesca de la amada.

\section{CONCLusiones}

A la luz de 10 antedicho, se pueden destacar los siguientes puntos:

- no son abundantes los objetos en el Idilio III, pero ayudan a complementar la vicisitud amorosa del cabrero.

- los objetos reales y concretos dentro del ámbito del poema son sólo dos: una guirnalda y la zamarra del pastor.

- la guirnalda forma parte de los accesorios que el amator llevaba consigo en el paraklausithyron y constituye un don amoroso que se colocaba en la puerta de la amada. En nuestro caso, ha sido trenzada por las manos del propio cabrero con plantas seleccionadas y, al parecer, será también el cabrero quien la destruirá. En el texto, la desaparición de la guirnalda anticipa, en la intención del protagonista, el motivo del suicidio.

- la zalea parece adquirir un valor peculiar en el ámbito bucólico. En este sentido, se convierte en un elemento que representa al personaje en su esencia. Salvarla de la destrucción o del "suicidio" es un modo de asegurar la fama póstuma de su dueño.

- el cedazo mágico anticipa el fracaso amoroso del cabrero, quien se revela como una personalidad supersticiosa ${ }^{29}$.

27. Theocr. I, 146; XX, 27.

28 . Theocr. V, 59.

29. Theocr. III, $28 \mathrm{ss}$. 
- la piedra - desde el punto de vista del cabrero - define, por su parte, a

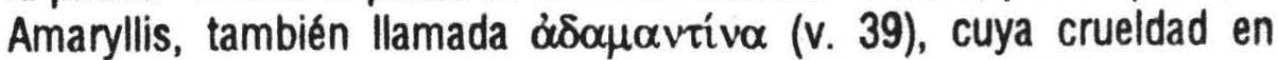
materia de amores, al final de la composición, es enfatizada para impacto del lector.

- la miel, siempre en la visión del hablante, aparece en un contexto metafórico de signo inverso al habitual por estar ligada a la idea de la muerte. En su fantasia, el cabrero finaliza "devorado" por Amaryllis, la loba entre los lobos, que representan la naturaleza salvaje y antibucólica en el habitat pacíficamente agreste de los pastores. Amor y Muerte concluyen por fusionarse en una única simbiosis que determina el destino final del protagonista.

Abstract: The third Idyll, which belongs to Theocritus' bucolic compositions, is at the same time a rustic paraklausithyron that has like protagonits an anonymous goatherd and an invisible shepherdess called Amaryllis. The essential subject deals, therefore, with love.

The present communication has been focalized exclusively from the level of the objects, an analysis prospect which is generally non common. The exam shows as a result that such objects are a few in the piece, but that all of them, rightly catalogued and analyzed, one by one, are intimately related to the main subject of the poem, and that whose study helps us to have a more complete view of the goatherd's love pains.

\section{Bibliografia}

SÁNCHEZ, M. B. Bucólicos griegos. Madrid, 1986.

GOW, A. Theocritus. Cambridge, 1952, 2 vol.

GALLAVOTII, C. Theocritus quique feruntur Bucolici Graeci. Roma 1946.

LEGRAND, P. H. Bucoliques Grecs. París 1927.

LAWALL, G. Theocritus' Coan Pastorals. Cambridge 1967.

SORIA, C. El $\pi \alpha \rho \alpha \kappa \lambda \alpha v \sigma i ́ \theta u p o v$ como presupuesto cultural de la elegía latina. REV. DE ESTUDIOS CLASICOS, T. VIII (1963), 55-94.

CANTER, $\boldsymbol{H}$. The paraclausithyron as a literary theme. AJPh 41 (1920), 355-368.

YARDLEY, J. The elegiac paraclausithyron. ERANOS 76 (1978), 19-34.

OT, U. Die Kunst des Gegensatzes in Theokrits Hirtengedichten. Hildesheim, 1969.

LEMBACH, K. Die Pflanzen bei Theokrit. Heidelberg, 1970.

RUMPEL, J. Lexicon Theocrite um. Hildesheim, 1961.

Sholia in Theocritum, Dübner, Parisii, Ed. Ambrosio Firmin-Didot, 1878.

ARNOTT, G. Coscinomancy in Theocritus and Kazantzakis. MNEM. 31, Fasc. n. 1 (1978), 27-32. 